\title{
In Vitro Antimicrobial Activity of Polyacrylamide Doped Magnetic Iron Oxide Nanoparticles
}

\author{
Munmun Mukherje
}

\begin{abstract}
This paper addresses the antimicrobial activity of cationic polymer doped iron oxide nanoparticles at in vitro level. Magnetic iron oxide nanoparticles were prepared in simple procedure and then doped with polyacrylamide (PAM). The antimicrobial activity of cationic polymer doped iron oxide nanoparticles was evaluated. The prepared polyacrylamide doped magnetic iron oxide nanoparticles exhibited antimicrobial activity against Escherichia coli. The study suggests that cationic polymer doped magnetic iron oxide nanoparticles have excellent bactericidal property and are useful in producing microbes free water.
\end{abstract}

Index Terms-Antimicrobial, cationic polymer, iron oxide nanoparticles, polyacrylamide.

\section{INTRODUCTION}

Microbiological contamination of water sources is a major concern worldwide. Research supports that, there are numbers of various bacterial species available (ranging from 102 to $104 \mathrm{~mL}^{-1}$ ) in raw water as well as sewage effluents [1]. Microbiological contamination in any sources should be avoided at any cost since in the production of potable water, only a limited number of bacteria (depends on the type of bacteria) are acceptable. The process for the removal of contaminants depends not only on the nature of the microorganisms but also on the desired levels of purity.

Magnetic iron oxide nanoparticles $\left(\mathrm{Fe}_{3} \mathrm{O}_{4}\right)$ are common ferrite that has a cubic inverse spinel structure. The compound exhibits unique magnetic properties based on the transfer of electrons between $\mathrm{Fe}^{2+}$ and $\mathrm{Fe}^{3+}$ in the octahedral sites. Synthesis of magnetite $\left(\mathrm{Fe}_{3} \mathrm{O}_{4}\right)$ nanoparticles has long been of great interest because of their immense usefulness in different domains, especially in the form of ferro-fluids. The use of advanced nanotechnology in water purification for the production of safe drinking water is being currently investigated. Nanotechnology, the deliberate manipulation of matter at size scales of less than $100 \mathrm{~nm}$, holds the promise of creating new materials and devices which take advantage of unique phenomena realized at those length scales, because of their high reactivity due to the large surface-to-volume ratio [2]-[5].

It has been reported in many previous published work that, cationic polymers can increase the settling rate of bacterial floc and improve capture of dispersed floc, suspended solids and cell fragments. Usually, cationic polymers may also be used on a temporary basis to improve the removal of undesirable organisms, such as filamentous bacteria or yeast infestations that cause sludge bulking or carryover of floating clumps of sludge [6]. 2-hydroxy propyl dimethyl ammonium chloride, pyrrole-2, 3-dicarboxylic acid (PDCA) are of great interest due to their potential in water purification such as sludge dewatering, treatment of municipal and industrial waste water etc. Polyacrylamide (PAM) is commercially important cationic polymer for water treatment due to its high efficiency and rapid dissolution property [7].

In this paper the antimicrobial activity of cationic polymer doped iron oxide nanoparticles was evaluated in order to deduce its dose and efficacy on Escherichia coli strain.

\section{EXPERIMENTAL SETUP}

\section{A. Experimental Materials}

Polyethylene glycol, polyacrylamide (PAM), ferrous sulphate (heptahydrate), ammonia solutions (25\%), nutrient agar ( $\mathrm{pH}$ 7.0) was purchased from M/s, Merck (India) Mumbai Ltd. Ferric chloride (hexahydrate) was purchased from Lobachemie, India.

\section{B. Synthesis of Magnetic Iron Oxide Nanoparticles $\left(\mathrm{Fe}_{3} \mathrm{O}_{4}\right)$}

Typically $2.00 \mathrm{~g}$ of ferric chloride $\left(\mathrm{FeCl}_{3}\right)$ and ferrous sulphate $\left(\mathrm{FeSO}_{4}\right)$ were dissolved into $50 \mathrm{ml}$ of distilled water. To this ammonium hydroxide was added under vigorous stirring. Polyethylene glycol was quickly added into the solutions as surfactant. The resultant mixture is heated at $80^{\circ} \mathrm{C}$ until the black precipitate was appeared. The black precipitate was then thoroughly washed several times with distilled water and then with acetone. The magnetic nanoparticles were then dried at room temperature.

\section{Synthesis of Polyacrylamide Doped Iron Oxide Nanoparticles $\left(\mathrm{Fe}_{3} \mathrm{O}_{4}\right)$}

To modify synthesized magnetic iron oxide nanoparticles with polyacrylamide, magnetic iron oxide nanoparticles $\left(\mathrm{Fe}_{3} \mathrm{O}_{4}\right)$ and polyacrylamide $(1: 1)$ were first suspended in 30 $\mathrm{ml}$ distilled water and stirred for 30 minutes for maximum dissolution. The resultant solution was then used without further modification.

Next we describe the experimental evaluation of the in vitro antimicrobial effects of polyacrylamide doped magnetic iron oxide $\left(\mathrm{Fe}_{3} \mathrm{O}_{4}\right)$.

\section{IN VITRO ANTIMICROBIAL EFFECTS}

Munmun Mukherjee is with the Department of Chemical Engineering, Indian Institute of Technology, Kharagpur, West Bengal, India-721302 (e-mail: mukherjee.munmun@ gmail.com).
Multiple tube fermentation method was applied to study 
the antimicrobial property of magnetic nanopolymer. The minimum inhibitory concentration (MIC) of magnetic nanopolymer was determined with fresh Escherichia coli culture grown in laboratory. EC (Escherichia coli) broth was freshly prepared and poured into ten test tubes $(10 \mathrm{ml} / \mathrm{test}$ tube). These test tubes containing culture medium was then autoclaved at $121^{\circ} \mathrm{C}$ for 15 minutes (15 lb pressure). The bacteria culture (Escherichia coli) was diluted with sample water and vortexed until it reached 100-110 CFU/ml. The resultant sample water was then poured into different conical flasks. Various dosages of polyacrylamide doped iron oxide nanoparticles were introduced into the flasks $(50-150 \mu \mathrm{l})$ and mixed carefully. The conical flasks were then shaken in an incubator for 24 hours at $37^{\circ} \mathrm{C}$. An inoculum of resultant sample was introduced to each test tube containing EC broth (Escherichia coli) and mixed carefully with shaking. The test tubes were immediately incubated for 24-36 hours at $37^{\circ} \mathrm{C}$. Appearance of turbidity or gas production indicated the growth of Escherichia coli in the culture tube. The lowest concentration (dose) of polyacrylamide doped magnetic iron oxide nanoparticles that completely inhibited the growth of E. coli (indicated by no visible turbidity in the test tube) was recorded.

\section{CHARACTERIZATION OF NANOPARTICLES}

The phase analysis of the synthesized magnetite nanopowder was performed on an X'pert Pro Phillips X-ray diffractometer (XRD). High-resolution transmission electron microscopy (JEOL 3010, Japan) was employed to characterize the microstructure of the nanoparticles. The nanoparticles were thoroughly dissolved in distilled water and a drop of the solution was placed on the copper grid. The size and $\xi$ potential of the nanoparticles were measured after complete dissolution of nanoparticles in distilled water in the laboratory, by particle size analyzer (DTS Ver. 5.00; Malvern Instruments Ltd.). The presence of surface functional groups was investigated by Fourier Transform Infrared (FTIR) spectroscopy by PerkinElmer Spectrum 100 spectrommeters, USA. The sample was dried and FTIR study was done in the range of 400-4000 $\mathrm{cm}^{-1}$. Magnetic property of the prepared nanoparticles was investigated by SQUID-VSM instrument (Evercool SQUID VSM DC Magnetometer).

\section{RESULTS AND DISCUSSIONS}

\section{A. Nanoparticle Size and Surface Charge}

The transmission electron micrograph of magnetite nanoparticles, presented in Fig. 1(a) shows that particles have spherical morphology with some agglomeration, which is obvious because of magnetic dipolar interactions. The size of the magnetic nanoparticles was 10-20 nm. The surface of iron oxide nanoparticles was found to be negatively charged $(-20 \mathrm{mV})$, as evident from the results in Fig. 1(b).

\section{B. X-Ray Analysis}

The high-resolution $x$-ray diffraction pattern of iron oxide magnetic nanoparticles has been shown in Fig. 1(c). The $d$ values correspond to those of spinel phase of magnetite
$\left(\mathrm{Fe}_{3} \mathrm{O}_{4}\right)$. The broadening of XRD peaks indicates the nanocrystalline nature of the synthesized magnetite nanopowder. Taking into account the broadening of each peak in XRD mean crystallite size was calculated by applying Scherrer's equation: $D=0.9 \lambda / \beta \cos \theta$, where $D$ is the average diameter in $\AA$, $\beta$ is the broadening of the diffraction line measured at half of its maximum intensity in radians, $\lambda$ is the wavelength of the $\mathrm{x}$-rays and $\theta$ is the Bragg diffraction angle. The mean crystallite size was found to be around $8 \mathrm{~nm}$.

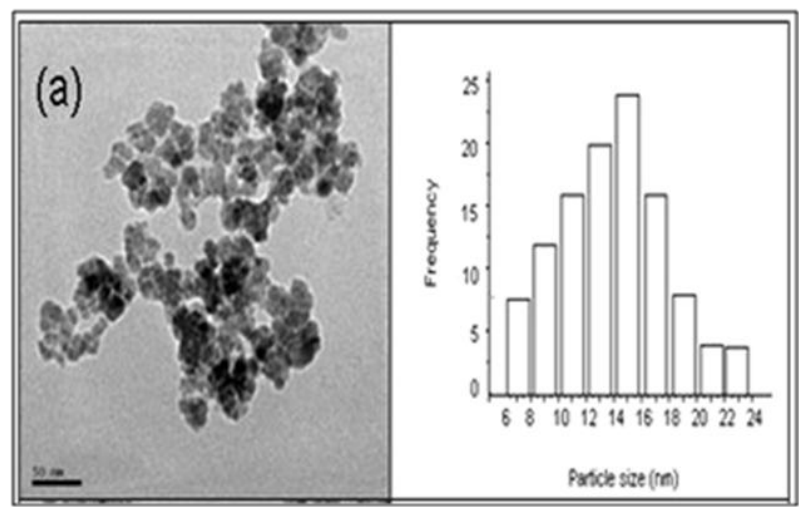

Fig. 1(a). TEM image of $\mathrm{Fe}_{3} \mathrm{O}_{4}$ nanoparticles.

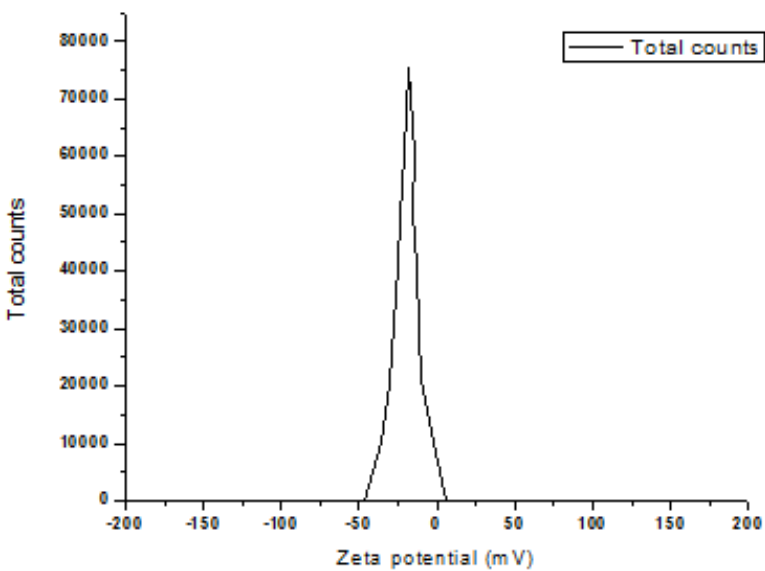

Fig. 1(b). Zeta potential of $\mathrm{Fe}_{3} \mathrm{O}_{4}$ nanoparticles.

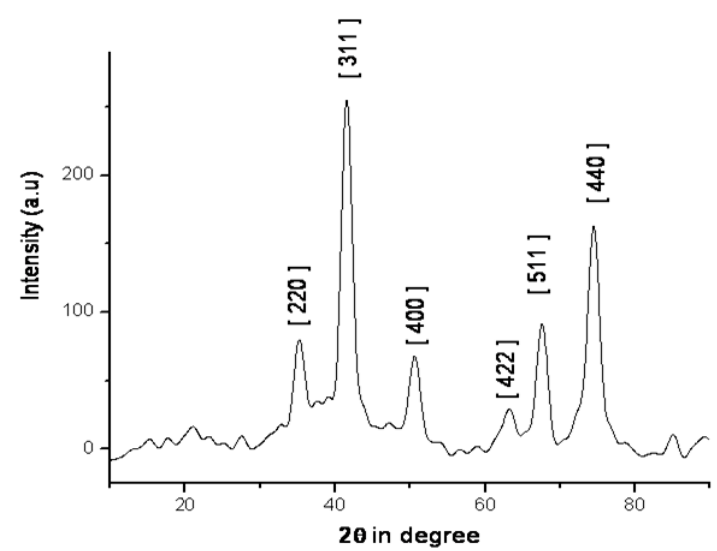

Fig. 1(c). XRD of $\mathrm{Fe}_{3} \mathrm{O}_{4}$ nanoparticles.

\section{FTIR Analysis}

The FTIR study of iron-oxide nanoparticles show a broad band around $3399 \mathrm{~cm}^{-1}$, indicative of the presence of $-\mathrm{OH}$ groups on the nanoparticle surface. The strong band around $558 \mathrm{~cm}^{-1}$, characteristic of the $\mathrm{Fe}-\mathrm{O}$ vibration related to the 
magnetite core. A broad peak around $1666 \mathrm{~cm}^{-1}$ signifies presence of strong carbonyl group. However, the peak at $1632 \mathrm{~cm}^{-1}$ is due to the presence of C-C bond. Peaks at 1482 $\mathrm{cm}^{-1}$ and $1457 \mathrm{~cm}^{-1}$ are associated with $-\mathrm{C}-\mathrm{H}$ stretching. Bands at $1192 \mathrm{~cm}^{-1}$ are corresponding to C-O-C stretching vibrations Fig. 1 (d)),

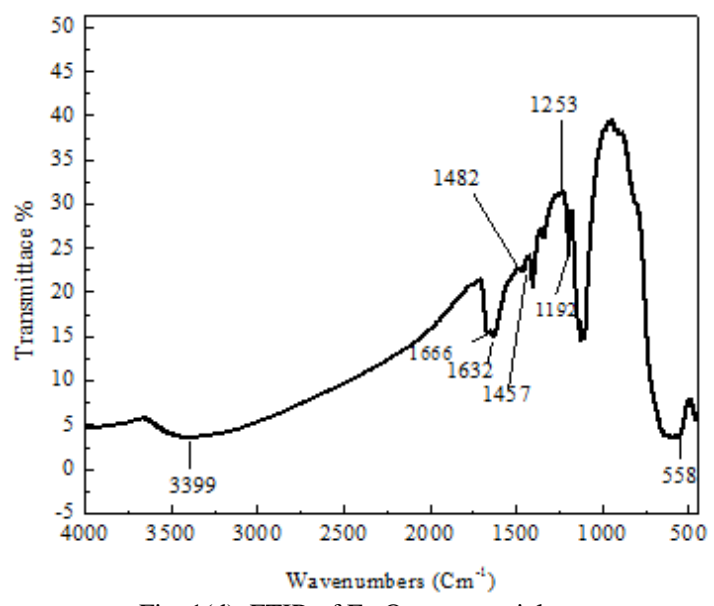

Fig. 1(d). FTIR of $\mathrm{Fe}_{3} \mathrm{O}_{4}$ nanoparticles.

\section{Magnetic Property}

The superparamagnetic properties of the magnetic nanoparticles is important to ensure their application in different fields, as it prevents them from aggregation and enables them to redisperse rapidly as soon as the magnetic field is detached. The magnetic property of invented iron oxide nanoparticles was measurements using SQUID-VSM instrument (Evercool SQUID VSM DC Magnetometer). The field dependent magnetization curve for the nanoparticles (Fig. 1(e)) showed no hysteresis and was completely reversible at $300 \mathrm{~K}$. Neither coercivity nor remanence was observed, indicating its superparamagnetic nature. The saturation magnetization $(M \mathrm{~s})$ was found to be $\approx 68 \mathrm{emu} \mathrm{g}^{-1}$, which is quite high.

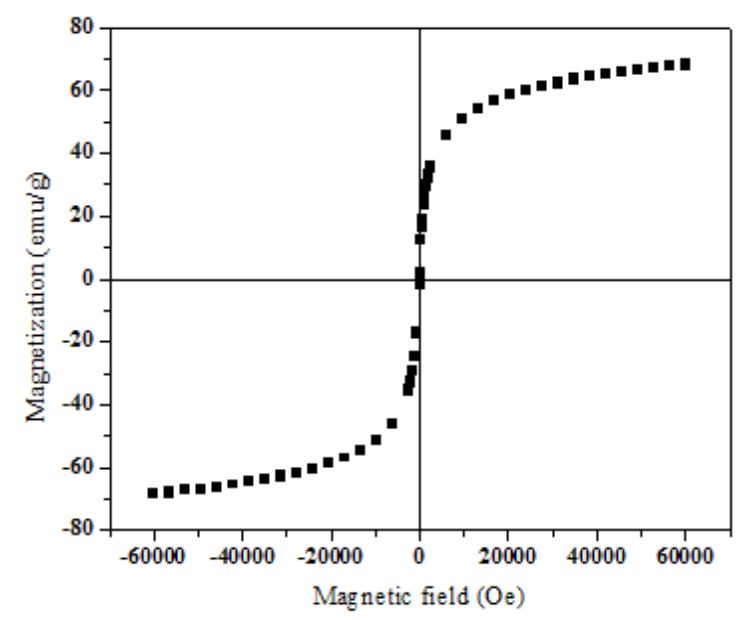

Fig. 1(e). Magnetic property of $\mathrm{Fe}_{3} \mathrm{O}_{4}$ nanoparticles.

\section{E. Antibacterial Activity of Polyacrylamide Doped Iron Oxide Nanopolymer}

Results show that minimum inhibitory concentration of polyacrylamide doped iron oxide were $60 \mu$ l that completely inhibited the growth of Escherichia coli. Iron oxide nanoparticles due to high ionic strength frequently agglomerate to some extent in environmental fluid or in the body before they reach their target. The nuclei of bacilli in the nanoparticles treated water sample culture seem to have ruptured due to incorporation of magnetic nanoparticles within the nuclei. The superoxide and hydroxide radicals of $\mathrm{Fe}_{3} \mathrm{O}_{4}$ seem to be the causative agent of such damage, as they may results in oxidative stress and consequent damage to proteins, membranes and DNA. The bond between superoxide radical of $\mathrm{Fe}_{3} \mathrm{O}_{4}$ and $\mathrm{NH}_{2}$ group of polyacrylamide enhanced antimicrobial activity of the resultant nanopolymer. Thus, growth of Escherichia coli completely inhibited as MIC was achieved. This finding is directly corroborated by earlier research publications [7].

\section{INFERENCE}

In brief, highly monodispersed, magnetic iron oxide nanoparticles (10-20 nm) modified with polyacrylamide has been produced using simple technique and low cost, non toxic chemicals. Polyacrylamide doped iron oxide nanoparticles showed excellent antimicrobial property.

The produced magnetic iron oxide nanoparticles are easily doped with polyacrylamide (PAM) and then experimented to figure out in vitro antimicrobial activity against Escherichia coli (100-110 CFU/ml) culture.

The results of the present study demonstrated that polyacrylamide doped iron oxide nanoparticles have bactericidal property and a minimum dose of $60 \mu \mathrm{l}$ completely inhibited the growth of Escherichia coli (100-110 $\mathrm{CFU} / \mathrm{ml}$ ).

In summary polyacrylamide doped iron oxide nanoparticles are safe to use as antimicrobial agent and hence can be used to produce microbe's free water as it showed high antimicrobial property.

\section{REFERENCES}

[1] D. S. Vicentini, A. Smania, and M. C. M. Laranjeira, "Chitosan/poly (vinyl alcohol) films containing $\mathrm{ZnO}$ nanoparticles and plasticizers," Mater. Sci. Eng. C-Mater. Biol. Appl., vol. 30 pp. 503-508, 2010.

[2] G. Li, Y. Jiang, K. Huang, P. Ding, J. Chen, "Preparation and properties of magnetic $\mathrm{Fe}_{3} \mathrm{O}_{4}$-chitosan nanoparticles," Journal of Alloys and Compounds, vol. 466, no. 5, pp. 451-456, 2008

[3] J. A. Lopez, F. González, F. A. Bonilla, G. Zambrano, M. E. Gómez, "Synthesis and characterization of $\mathrm{Fe}_{3} \mathrm{O}_{4}$ magnetic nanofluid," Rev. LatinAm. Metal. Mat., vol. 30, no. 1, pp. 60-66, 2010.

[4] T. Hyeon, "Large-scale synthesis of uniform and crystalline magnetite nanoparticles using reverse micelles as nanoreactors under reflux conditions," Adv. Funct. Mater. vol. 15, no. 3, pp. 503-509, 2005.

[5] S. Sun and H. Zeng, "Size-controlled synthesis of magnetite nanoparticles," Journal of the American Chemical Society, vol. 124, pp. 8204-8205, 2002.

[6] Handbook of Industrial Water Treatment, Water Treatment, Ch. 37.

[7] Dringking Water. [Online]. Available: http://www.snf-group.com/IMG/pdf/Drinking_Water_E.pdf

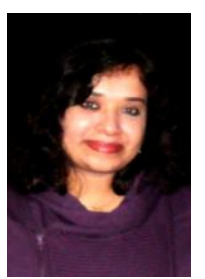

Munmun Mukherjee is from Kolkata, India. She completed M.Sc. in Environmental Science from Calcutta University in 2006. Earlier she did her B.Sc. in Zoology from Calcutta University in 2004. Her main research interests are synthesis and fabrication of various nanoparticles, water purification, sustainable agriculture etc. 